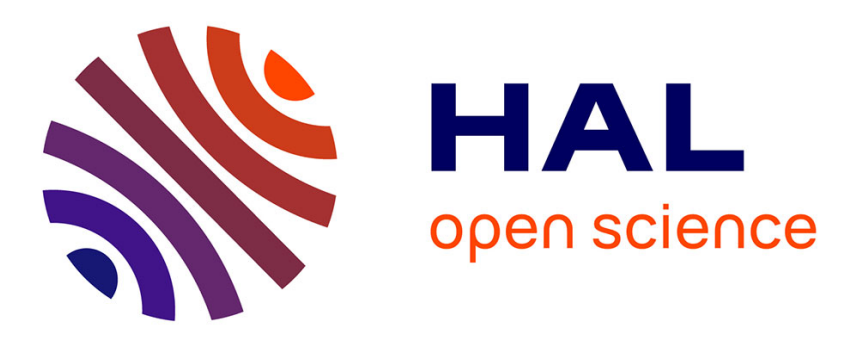

\title{
Toward a Time-centric modeling of Business Processes in BPMN 2.0.
}

Saoussen Cheikhrouhou, Slim Kallel, Nawal Guermouche, Mohamed Jmaiel

\section{To cite this version:}

Saoussen Cheikhrouhou, Slim Kallel, Nawal Guermouche, Mohamed Jmaiel. Toward a Time-centric modeling of Business Processes in BPMN 2.0.. 15th International Conference on Information Integration andWeb-based Applications \& Services (iiWAS'13), Dec 2013, Austria. pp.154. hal-00921390

\section{HAL Id: hal-00921390 \\ https://hal.science/hal-00921390}

Submitted on 13 May 2014

HAL is a multi-disciplinary open access archive for the deposit and dissemination of scientific research documents, whether they are published or not. The documents may come from teaching and research institutions in France or abroad, or from public or private research centers.
L'archive ouverte pluridisciplinaire HAL, est destinée au dépôt et à la diffusion de documents scientifiques de niveau recherche, publiés ou non, émanant des établissements d'enseignement et de recherche français ou étrangers, des laboratoires publics ou privés. 


\section{Toward a Time-centric modeling of Business Processes in BPMN 2.0}

\author{
Saoussen Cheikhrouhou \\ ReDCAD Laboratory \\ Univ. of Sfax, Tunisia \\ saoussen.cheikhrouhou \\ @redcad.org
}

\author{
Slim Kallel \\ ReDCAD Laboratory \\ Univ. of Sfax, Tunisia \\ slim.kallel@fsegs.rnu.tn
}

\author{
Nawal Guermouche ${ }^{1,2}$ \\ ${ }^{1}$ CNRS-LAAS, 7 avenue de \\ colonel Roche, F-31400 \\ Toulouse, France \\ ${ }^{2}$ Univ. of Toulouse, INSA, \\ LAAS, F-31400 Toulouse, \\ France \\ nawal.guermouche@laas.fr
}

\author{
Mohamed Jmaiel \\ ReDCAD Laboratory \\ Univ. of Sfax, Tunisia \\ mohamed.jmaiel@enis.rnu.tn
}

\begin{abstract}
Business-to-business (B2B) e-commerce market is expected to expand rapidly in coming years. In this context, organizations tend to rely more on business process management $(B P M)$ to streamline their operations. The business process field is influenced by a wide range of temporal constraints which rise from legal, regulatory, and managerial rules. One of the most promising standards for business process modeling, namely the Business process Model and notation BPMN poorly addresses the time dimension so far. In this paper, we elaborate an extension to BPMN 2.0 to handle the time dimension. The aim of this BPMN extensions is to support business analysts and modellers in easily including the needed temporal constraints in their processes. We motivate and justify our proposed extensions by means of illustrative case studies. Furthermore, based on the proposed extensions, a verification approach based on the model checking technique is used to diagnose potential temporal violations of the process model. The work presented in this paper sets foundation for later automation of these constraints through process execution engines.
\end{abstract}

\section{INTRODUCTION}

With the booming business globalization, organizations tend to rely more on business process management to streamline their operations. Such globalization trend highly urges collaborations among organisations forming thus a businessto-business e-commerce market. E-commerce market offers benefits to multiple entities, such as buyers, sellers and manufacturers. For buyers, it can significantly enhance the process of searching for providers, while for sellers it can provide access to a much broader customer base. Additionnally, it empowers the management of manufacturers' processes, which are outsourcing huge parts of their supply chains to outside contractors.

In this context, the business process field is influenced by a wide range of temporal constraints, which rise from legal, regulatory, and managerial rules. Obviously, the time perspective is a critical dimension to consider as it is closely related to customer satisfaction and cost reduction. The on-time delivery of goods or services has a direct impact on customer satisfaction. Furthermore, time management is often a very effective cost reduction strategy for organizations. Actually, business managers strive to have full support of temporal aspects in their business process management suites. Indeed, temporal constraints play a prevalent role in negociating delays of outsourcing, ensuring that tasks are completed on appropriate time and that the final product is ready within desired time. The time perspective is nevertheless lacking attention from current process standards initiatives. Considering the broadly accepted business process modeling language, BPMN, it poorly addresses the time dimension so far. This, in turn, has led to an increasing demand for innovative technologies and mechanisms that empower the time support from the business process modelling phase.

This paper proposes a BPMN 2.0 compliant extension to support the pecification of a large set of rich temporal constraints.

We intend to introduce additional modeling features to enable the specification of the different temporalities related to one activity as well as concurrent processes sharing resources and exchanging messages. For instance, we aim to address the different deadline constraints, the constraints related to the start and end events of the process activities, temporal constraints over cardinality, temporal points from the execution stage, etc.

These extensions will bring a multitude of benefits :

- Process models can easily be understood, shared and thus continuously improved.

- Process analysts can add the required temporal constraints to process models in a clear and unambiguous manner.

- Business experts can more efficiently specify and verify temporal constraints of the model.

The rest of this paper is structured as follows. We first identified some weakness of the BPMN standard in the specification of temporal constraints in Section 2. Section 3 
presents an example scenario which motivates and justifies the proposed extensions. Our proposed extentions to BPMN, are described in Section 4. Section 5 outlines the use of a formal verification approach to detect the temporal violations of process models. A review of related literature is given in Section 6. Finally, Section 7 concludes the paper.

\section{OVERVIEW OF THE BPMN LIMITATIONS IN THE SPECIFICATION OF TEMPORAL CONSTRAINTS}

One key perspective when dealing with Business Process Modelling is time. All business experts agree upon the fact that time is a key resource for each business process within an organisation. Indeed, processes have to meet certain deadlines and a certain coordination between process tasks must be achieved. The incorporation of the Timer Event in BPMN specification seems promising. Nevertheless, we argue that the Timer Event offered by the BPMN specification can represent the time dimension of processes, but only to a certain extend. Indeed, it is difficult to use the BPMN Timer Event to specify rich temporal consraints in an unambiguous manner which prevents the specification of processes where the time resource is relevant. The inefficiencies of BPMN to cope with the time perspective essentially appear in :

First, BPMN lacks for means to specify the turnaround time of business activities such as the minimum and maximum execution times [16]. Indeed, the use of the BPMN boundary Intermediate Event may lead to a misunderstanding of the intended temporalities. The BPMN boundary Intermediate Event is supposed to interrupt the corresponding activity rather than ensuring its completeness since it can only lead to an alternate flow [7]. Second, BPMN does not offer an explicit way to depict dependencies between more than one activity since it is not possible to add a temporal constraint on the end of an activity (task or sub-process) other than the default Finish-to-Start sequence flow. Third, in a business context, specifying the limited availability of some business activities is of paramount importance. Unfortunately, the BPMN specification lacks for means to specify delays within which activities must not occur. Fourth, BPMN does not sufficiently deal with temporal constraints correlated with resource constraints. Indeed, the resource perspective refers to the link between the defined activities and the entities that carry out the work related to them. The way in which the work is ultimately bound to specific resources is significant to the efficiency and effectiveness of the process. The limitations of BPMN in capturing the resource perspective stem from the Pool and Lane constructs. Indeed, these constructs poorly address the subtleties associated with selective work allocation across a range of possible resources [17] and the possibility of allocating more than one resource by the same activity.

This, in turn, has led to an increasing demand for extensions that address these limitations and elect time as a first time citizen in the business process modeling phase. Our main interest is to assist analysts and modellers in easily including the needed temporal constraints in their processes.

\section{MOTIVATING EXAMPLE}

Let us consider the BPMN diagram of the purchase order process in a manufacturing organisation depicted in Fig. 1.

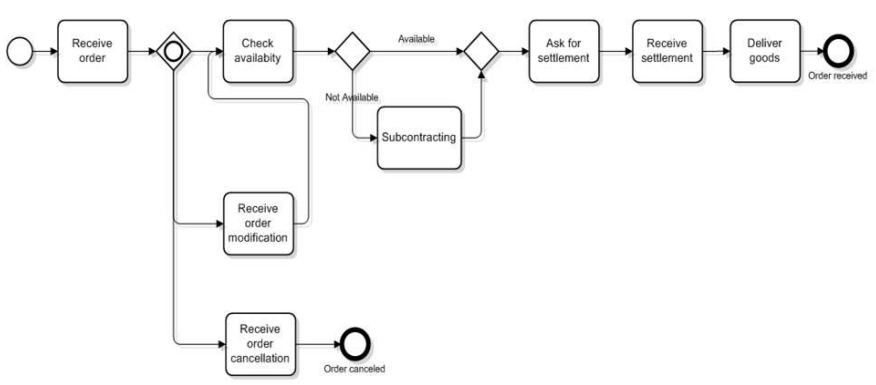

Figure 1: A BPMN process diagram of the purchase order handling

The process is triggered when a customer submits a purchase order (Receive order). Then, it can check whether the ordered articles are available or not (Check availability), modify (Receive order modification) and even cancel the requested order (Receive order cancellation). In case of order modification, the availability of the order is checked again. If the ordered articles are available in stock, the customer is asked for financial settlement (Ask for settlement and Receive settlement) and the goods are subsequently delivered (Deliver goods), otherwise the organisation needs to launch a subcontracting activity (Subcontracting). By the reception of an order cancellation, the process meets its end.

Within business processes, the temporal perspective is crucial since temporal constraints must be respected. For instance, we can mention the following constraints:

- constraint 1: During processing time it may be possible for the customer to cancel an order, but once a subcontracting activity is triggered, the order cannot be cancelled anymore,

- constraint 2: The activity Deliver goods of the purchase order handling process have to start no later than $22 \mathrm{~h}$ once the process starts,

- constraint 3: The manufacturing organisation can not receive orders in the evening (from $22 \mathrm{~h}-8 \mathrm{~h}$ ),

- constraint 4: If an order cancellation exists, it should be finished before the subcontracting activity begins, and

- constraint 5: Order modification is only allowed maximum 1 day after the order was received .

BPMN allows to specify some kind of temporal constraints. For example, constraints 2 which states that the activity Deliver goods of the purchase order handling process have to start no later than 22h once the process starts can be specified as shown in Fig. 2. It is appropriate to emphasize the complexity of the BPMN notation to depict constraint2 as well. It is clear that tackling such temporal constraints with BPMN can be overwhelming without a simplified extension.

In spite BPMN offers some capabilities to specify temporal constraints, it remains very poor and does not allow to capture a wide range of temporal constraints. For example, it is not possible to specify the temporal perspective presented in constraint1 using the BPMN notation, since the 


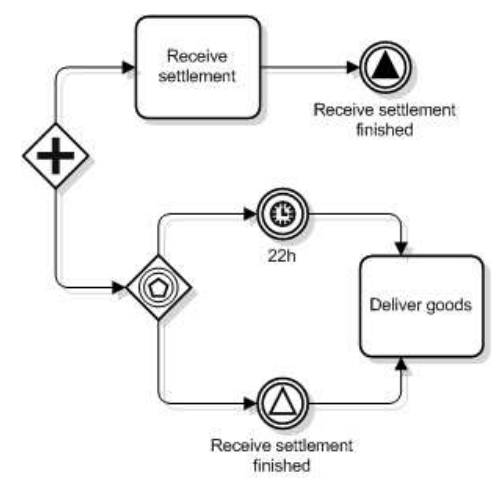

Figure 2: A BPMN diagram to depict constraint2

latter standard does not allow the depiction of dual temporal dependencies between two activities [6].

Failing to include rich temporal constraints in process models turns out in higher process execution costs, either by loss of productivity, lack of coordination, or missed deadlines committed with the involved customers. In the following, we describe the proposed BPMN extension for capturing rich temporal constraints during business process modelling.

\section{EXTENDED BPMN NOTATION TO SUP- PORT THE TEMPORAL PERSPECTIVE}

This section is devoted to give an overview of the basic process modelling concepts of the proposed BPMN extension to well support the temporal perspective in process models. We distinguish three major categories of temporal constraints:

1. Intra-activity temporal constraints

2. Inter-activity temporal constraints

3. Inter-process temporal constraints

4. Temporal constraints correlated with resource/data constraints

Hereafter, we describe each category.

\subsection{Intra-activity temporal constraints}

This category gathers temporal constraints (TC) associated to one activity within a business process such as : (1) duration, (2) TC over cardinality, (3) start/end TC, and (4) the intra activity absence constraint.

Note : As per BPMN, we treat time values (i.e. time points and durations) as Expression (see the BPMN specification [2] p.274).

\subsubsection{Duration:}

Given the limits of BPMN to specify the turnaround time of business activities, an activity decorator with the minimum and maximum duration values is proposed and respectively depicted by the label 1 in Fig.3.

The duration constraint can be specified for process activities (task or sub-process). The attributes of the duration constraints are described in table 1.

To precise the execution semantic of this constraint, we propose to add the attributes isInterrupting and is Awaiting.
Table 1: Duration constraint attributes

\begin{tabular}{|l|l|}
\hline Attribute & Description \\
\hline Id & unique and required for identification \\
\hline Name & optional \\
\hline Target & $\begin{array}{l}\text { required and unique. The target must be } \\
\text { an activity }\end{array}$ \\
\hline Min_Time & $\begin{array}{l}\text { (specified as a BPMN Expression) required } \\
\text { and is the minimum duration value of the } \\
\text { activity }\end{array}$ \\
\hline Max_Time & $\begin{array}{l}\text { (specified as a BPMN Expression) required } \\
\text { and is the maximum duration value of the } \\
\text { activity }\end{array}$ \\
\hline
\end{tabular}

If a given activity, say A1, has a duration constraint with the attribute is Awaiting set to true, and it attempts to finish its execution before the duration mentioned in the Min_Time attribute, the process engine has to wait for the minimum execution time to elapse before reaching the suceeding activity, say A2. If the attribute isInterrupting is set to true, the execution of the task trying to exceed the duration precised in its Max_Time attribute must be interrupted, as well.

\subsubsection{Temporal constraint over cardinality:}

This temporal constraint denotes that an activity can be executed successively $n$ times whithin a time period. The BPMN standard already provides constructs to specify such constraint (i.e. A loop activity with an Intermediate Event attached to its boundary can be used for that purpose). We use the BPMN notation depicted by label 2 in Fig.3 to specify that during 15 minutes, a customer can only do 3 failed payment trials. This constraint helps to manage security in process aware information systems.

\subsubsection{Start/End Temporal Constraints :}

The succeeding listing summarizes the proposed temporal constraints to control start and finish times of process activities.

- Must Start On (MSO), Must Finish On (MFO)

- Start As Soon As Possible (SASAP), Finish As Soon As Possible(FASAP)

- Start No Earlier Than (SNET), Finish No Earlier Than (FNET), and

- Start No Later Than (SNLT), Finish No Later Than (FNLT).

Fig. 4 outlines the proposed Start/Finish No Later Than (SNLT/FNLT) and the Must Start/Finish On (MSO/MFO) temporal constraints.

The figures of the rest of Start/End TC are mainly inspired from the Time-BPMN [7] and are omitted due to space limitation.

Start and end temporal constraints can be specified for process activities (task or sub-process). The attributes of the start or end temporal constraints are described in table 2 .

\subsubsection{The intra-activity absence constraint :}

To specify delays within which activities must not occur, we propose to extend BPMN by the Absence decorator attached to the boundary of activities. The temporal constraint, denoted by the label $\mathbf{3}$ in Fig. 3 depicts a constraint which states that the manufacturing organisation can not re- 


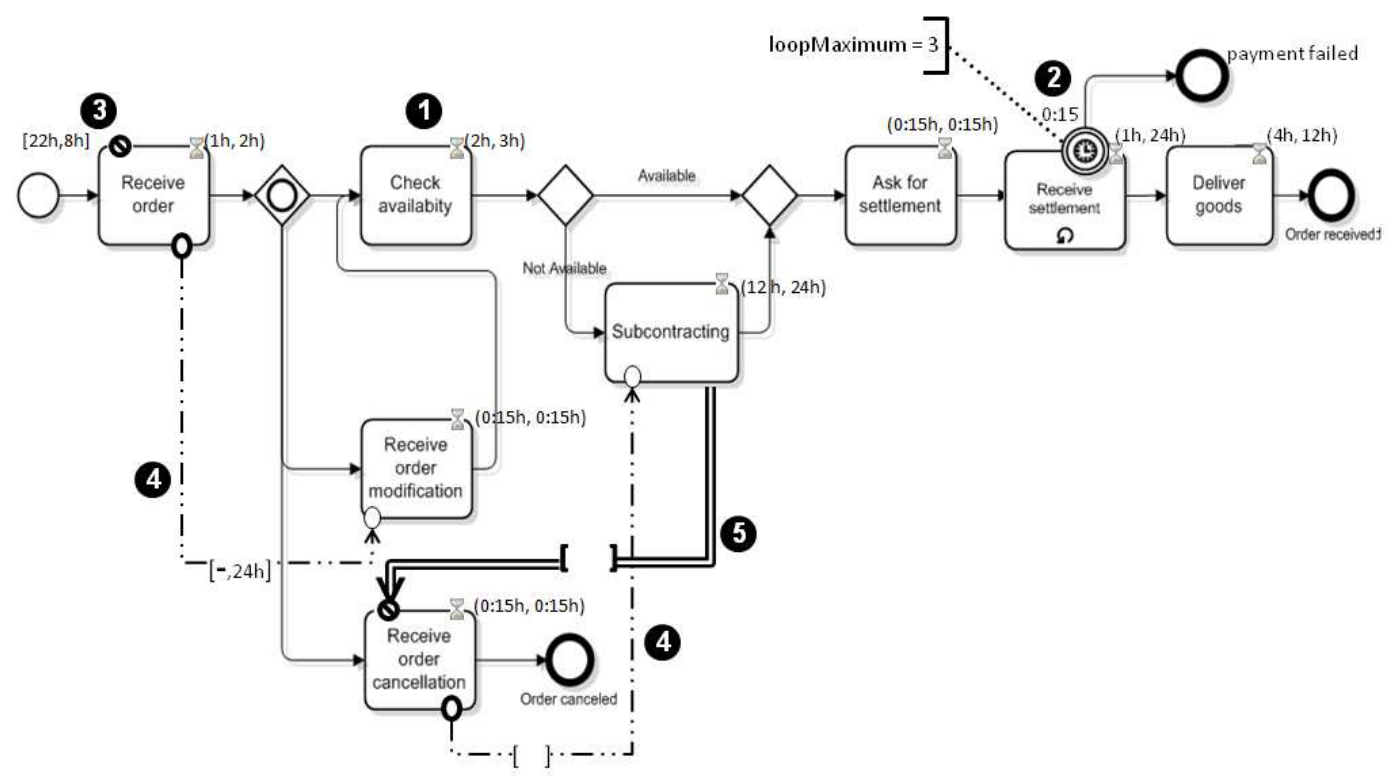

Figure 3: Extended BPMN diagram of the purchase order handling proces

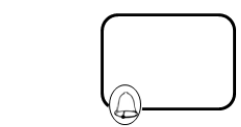

Start No Later Than (SNLT)

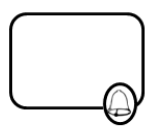

Finish No Later Than (FNLT)

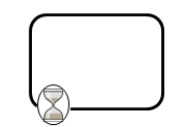

Must Start On (MSO) Must Finish On (MFO)

Figure 4: Proposed BPMN extensions to model some proposed temporal constraints

Table 2: Start/end temporal constraints attributes

\begin{tabular}{|l|l|}
\hline Attribute & Description \\
\hline Id & unique and required for identification \\
\hline Name & optional \\
\hline Target & $\begin{array}{l}\text { required and unique. The target can be an } \\
\text { activity or a Resource-Group (see Section } \\
4.4)\end{array}$ \\
\hline Type & $\begin{array}{l}\text { required. It indicates the type of start } \\
\text { or end temporal constraint wether } \\
\text { it is MSO/MFO, SASAP/FASAP, } \\
\text { SNET/FNET or SNLT/FNLT. It is } \\
\text { relative to a point in time as specified in } \\
\text { the Value attribute described below }\end{array}$ \\
\hline Value & $\begin{array}{l}\text { required and specified as a BPMN Expres- } \\
\text { sion }\end{array}$ \\
\hline
\end{tabular}

ceive orders in the evening (from 22h-8h) due to the availability of some workers for example. We propose hence a solution to the depiction of constraint 3 of Section 2 .

The intra-activity absence constraint can be specified for process activities (task or sub-process). The attributes of the intra-activity absence constraint are described in table 3.

\subsection{Inter-activity temporal constraints}

This subsection is devoted to temporal constraints crossing the boundary of an activity in the Process Model such as : (1) temporal dependency, and (2) inter-activity absence constraint.
Table 3: The intra-activity absence constraint attributes

\begin{tabular}{|l|l|}
\hline Attribute & Description \\
\hline Id & unique and required for identification \\
\hline Name & optional \\
\hline Target & $\begin{array}{l}\text { required and unique. The target must be } \\
\text { an activity }\end{array}$ \\
\hline From_Value & $\begin{array}{l}\text { specified as a BPMN Expression. It spec- } \\
\text { ifies the minimum value of the interval in } \\
\text { which the corresponding activity must be } \\
\text { absent }\end{array}$ \\
\hline To_Value & $\begin{array}{l}\text { specified as a BPMN Expression. It spec- } \\
\text { ifies the maximum value of the interval in } \\
\text { which the corresponding activity must be } \\
\text { absent }\end{array}$ \\
\hline
\end{tabular}

\subsubsection{Temporal Dependency:}

A temporal dependency is a relationship between two activities, say $A_{1}$ and $A_{2}$, in which one activity depends on the start or finish of another activity in order to begin or end. The temporal dependencies suggested in this paper enhance the expressiveness of previous proposals in representing such dependencies. For instance, Time-BPMN [7] assigns lead and lag times to a temporal dependency relations. In our work, we propose a fine grained temporal dependencies. Indeed, we propose to use intervals to precise the minimum and maximum time bounds of temporal dependencies. We propose the following four temporal dependencies: 
- Start-to-Finish (SF): $A_{2}$ can not finish until $A_{1}$ has started within a given time interval

- Start-to-Start (SS) : $A_{2}$ can not begin before $A_{1}$ starts within a time interval

- Finish-to-Start (FS) : $A_{2}$ can not begin before $A_{1}$ ends within a time interval

- Finish-to-Finish (FF) : $A_{2}$ can not finish until $A_{1}$ has finished within a time interval

Let us consider the two constraints (4) and (5) presented in section 2 which state respectively:

- If an order cancellation exists, it should be finished before the subcontracting activity begins

- Order modification is only allowed maximum 1 day after the received order

Using the temporal dependencies presented above, these two constraints can be specified as shown by label $\boldsymbol{4}$ in Fig. 3.

The temporal dependency constraint can be specified for process activities (task or sub-process). The attributes of the temporal dependency constraint are described in table 4 .

Table 4: The temporal dependency constraint attributes

\begin{tabular}{|l|l|}
\hline Attribute & Description \\
\hline Id & unique and required for identification \\
\hline Source & $\begin{array}{l}\text { required and unique. The source must be } \\
\text { an activity }\end{array}$ \\
\hline Target & $\begin{array}{l}\text { required and unique. The target must be } \\
\text { an activity }\end{array}$ \\
\hline From_Value & $\begin{array}{l}\text { specified as a BPMN Expression. It spec- } \\
\text { ifies the minimum value of the interval of } \\
\text { the temporal dependency constraint }\end{array}$ \\
\hline To_Value & $\begin{array}{l}\text { specified as a BPMN Expression. It spec- } \\
\text { ifies the maximum value of the interval of } \\
\text { the temporal dependency constraint }\end{array}$ \\
\hline
\end{tabular}

\subsubsection{The inter-activity absence constraint:}

This kind of constraints enables specifying delays within which activities must not occur. For instance, to specify constraint 1 of Section 2 which denotes that the order can not be cancelled after the beginning of a subcontracting activity is not possible in BPMN. To tackle such feature, we propose the BPMN extension denoted by the label $\mathbf{5}$ in Fig. 3.

The inter-activity absence constraint can be specified for process activities (task or sub-process). The attributes of the inter-activity absence constraint are described in table 5.

\subsection{Inter-process temporal constraints}

In this subsection, we introduce temporal constraints crossing the boundary of one process such as : (1) exchanged temporal data and (2) deadline of message exchange.

\subsubsection{Exchanged Temporal Data:}

In this subsection, we lay great stress upon the need to distinguish messages with temporal data from other kind of data from the specification step. The goal is the consequent use of these temporal data to specify rich tempo-
Table 5: The inter-activity absence constraint attributes

\begin{tabular}{|l|l|}
\hline Attribute & Description \\
\hline Id & unique and required for identification \\
\hline Name & optional \\
\hline Source & $\begin{array}{l}\text { required and unique. The source must be } \\
\text { an activity }\end{array}$ \\
\hline Target & $\begin{array}{l}\text { required and unique. The target must be } \\
\text { an activity }\end{array}$ \\
\hline Value & $\begin{array}{l}\text { specified as a BPMN Expression. It speci- } \\
\text { fies the duration in which the target activ- } \\
\text { ity must be absent after the ending of the } \\
\text { source activity }\end{array}$ \\
\hline
\end{tabular}

ral constraints among the process activities. Once temporal data are well specified, their circulation and negociation between organisations would be much easier. This would be inevitable to clearly model these temporal data, especially when these organisations are involved in an interorganisational business process. Temporal data sent by an organisation, say O1, to another organisation, say O2, must be used to constrain some activities in the process model of O2. Hence, the message flow label $\mathbf{T}$ (delivery_date) presented in Fig. 5 is the proposed notation to insist on the fact that delivery_date is a temporal information.

To add this latter extension, we propose to add the attribute is TemporalData to the BPMN message attributes (see the BPMN specification [2] p.95) . The attribute is TemporalData set to true means that the corresponding message presents a temporal information.

\subsubsection{Deadline of message exchange :}

In an inter-organizational context, allowing to impose constraints on messages exchanged between the involved partners seems to be necessary. For instance, $48 \mathrm{~h}$ of delay is specified between the messages presented in Fig. 5.

The attributes relating to the constraint deadline of message exchange are described in table 6 .

Table 6: The deadline of message exchange attributes

\begin{tabular}{|l|l|}
\hline Attribute & Description \\
\hline Id & unique and required for identification \\
\hline Name & optional \\
\hline Source & $\begin{array}{l}\text { required and unique. The source must be } \\
\text { a BPMN message }\end{array}$ \\
\hline Target & $\begin{array}{l}\text { required and unique. The target must be } \\
\text { a BPMN message }\end{array}$ \\
\hline Value & $\begin{array}{l}\text { specified as a BPMN Expression. It speci- } \\
\text { fies the delay to be respected between two } \\
\text { exchanged messages }\end{array}$ \\
\hline
\end{tabular}

\subsection{Temporal Constraints correlated with re- source /data constraints}

Our work comes up with other temporal constraints such as the ones correlated with resource and data constraints.

\subsubsection{TC correlated with resource constraints :}




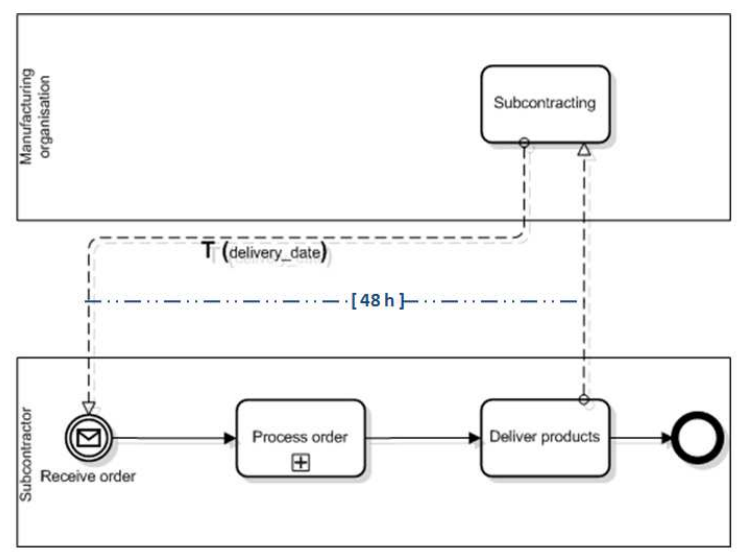

Figure 5: Exchanged temporal data and the deadline of message exchange temporal constraints

In order to introduce temporal constraints correlated with resource constraint in $\mathrm{BPMN}$, we introduce an additional construct called Resource-Group as a rounded corner rectangle with a solid dashed line marked with "R" to indicate that the inner activities/processes compete for the same indicated resource. On top of it, the name of the resource is precised. To give emphasis to the availability of resources, we propose the use of the already proposed Start/End temporal constraints for the Resource-Group as well. The ResourceGroup has two attributes named Allocation_policy and Quantity wich stand for the allocation policy (eg. the first-comefirst-served(FCFS) policy or short processing time (SPT)) and the number of required resources respectively. Imbricated Resource-Group can be used to mention that the inner activities use more than one resource. Consider the Receiving station process depicted in Fig. 6. The execution of the inner activities (record parts documentation in the system and record boxes location change in the system) requires the availability of both resources : a technician as a human resource and a RMS (i.e. Records Management system) as system resource.

The Resource-Group can be specified as a BPMN element. The attributes of the Resource-Group are described in table 7 .

Table 7: The Resource-Group attributes

\begin{tabular}{|l|l}
\hline Attribute & Description \\
\hline Id & unique and required for identification \\
\hline Name & optional \\
\hline Activity & required \\
\hline
\end{tabular}

\subsubsection{TC correlated with data constraints :}

Temporal constraints correlated with data constraints are an important issue to consider when specifying real business scenarios. For instance, when considering processes of perishable products suppliers, the duration of the $\mathrm{Ma}$ terial's cold storage activity depends heavily on the recommended temperature of the product. The tabular depicted in Fig. 7 shows our BPMN extension to specify that the $d u$ ration of the Material's cold storage activity is between $1 \mathrm{~h}$ and $2 h$ if the product's recommended temperature temp is lower than $15^{\circ} \mathrm{C}$ (i.e. temp $\leq 15^{\circ} \mathrm{C}$ ) and between $2 \mathrm{~h}$ and $4 \mathrm{~h}$ otherwise. The proposed notation is light-weighted and can be collapsed (see Fig. 7(a)) or expanded (see Fig. 7(b)) as appropriate.

The temporal constraints correlated with data constraints can be specified for process activities (task or sub-process). The attributes of the TC correlated with data constraints are described in table 8 .

Table 8: TC correlated with data constraints attributes

\begin{tabular}{|c|c|}
\hline Attribute & Description \\
\hline $\mathrm{Id}$ & unique and required for identification \\
\hline Name & optional \\
\hline Target & $\begin{array}{l}\text { required and unique. The target must be } \\
\text { an activity }\end{array}$ \\
\hline Condition1 & $\begin{array}{l}\text { (specified as a BPMN Expression) required } \\
\text { and is the condition to set Value } 1 \text { as a du- } \\
\text { ration for the activity mentioned in the tar- } \\
\text { get attribute }\end{array}$ \\
\hline Value1 & (specified as a BPMN Expression) required \\
\hline Condition2 & $\begin{array}{l}\text { (specified as a BPMN Expression) required } \\
\text { and is the condition to set Value } 2 \text { as a du- } \\
\text { ration for the activity mentioned in the tar- } \\
\text { get attribute }\end{array}$ \\
\hline Value2 & (specified as a BPMN Expression) required \\
\hline
\end{tabular}

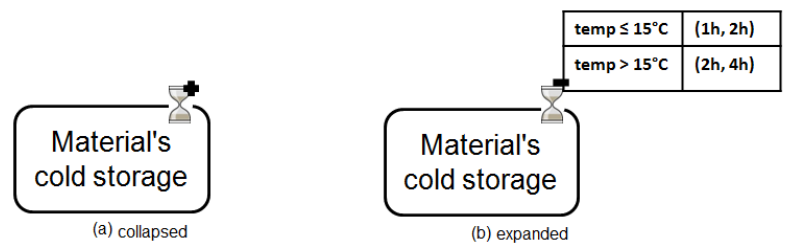

Figure 7: Temporal constraint correlated with data constraints

\subsection{Prototype}

Currently, we are working on a tool support for the presented BPMN extensions. We are implementing a prototype extending the Activiti Eclipse Designer (http://www.activiti 


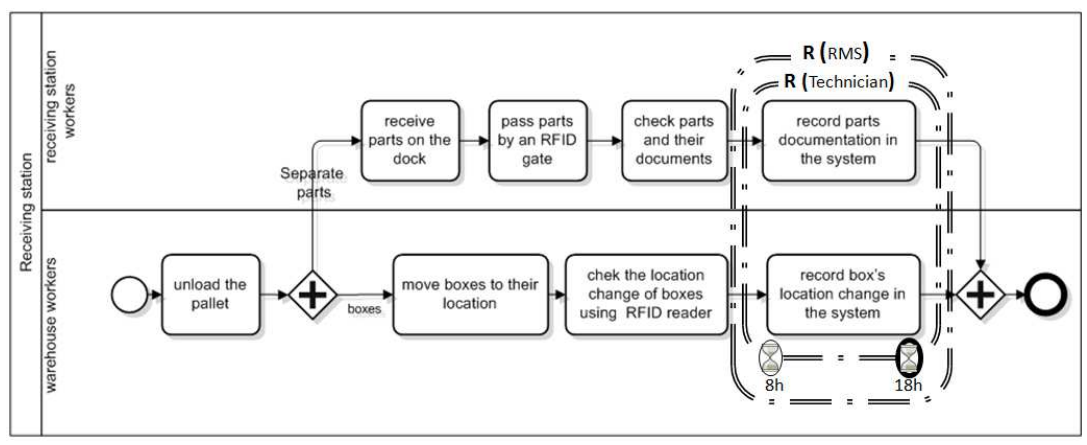

Figure 6: Temporal constraint correlated with resource constraints

.org/). The Activiti BPM platform is a simple, lightweight, and open-source business process management platform. Some of the appealing features of Activiti include its BPMN 2.0 based process engine. The screenshot of the prototype is shown in Fig. 8.

\section{THE VERIFICATION FRAMEWORK}

Considering temporal constraints while specifying process models improves expressiveness and opens the way to the integration of formal validation tools. Indeed, based on the proposed extensions, a verification approach based on the model checking technique is used to diagnose potential temporal violations of the model. Our approach allows the verification of deadlock freedom. Moreover, it enables the designer to check user-defined temporal constraints such as deadlines.

We argue that temporal verification mechanisms are of paramount importance since they enable to detect, early on, possible temporal conflicts and to react to them effectively.

Precisely, the designer can verify delays between two activities $A_{i}$ and $A_{j}$ of a process or between the start of the process and its end. We are convinced that it is not enough to verify processes separately. Indeed, It is also worthwhile to consider temporal violations occuring during the collaboration of multiple processes.

For the formal verification of processes, we use the UPPAAL model checker. UPPAAL is devoted to verify realtime systems modelled as networks of timed automata (TA). A timed automaton has invariants on the locations, and has actions, guards, and assignments on the edges, used for restricting its behavior. The query language used to specify properties to be checked by Uppaal, is a subset of CTL (computation tree logic).

Timed automata models have been the basis of a wide range of successful verification methodologies allowing the efficient prediction of time-related properties(e.g. absence of deadlock or deadline constraints).

Thus, as a first step, processes are mapped onto timed automata. The mapping used in this work builds upon our previous work $[8,4]$.

To show the applicability of our verification approach, we consider a simplified process diagram of the already defined purchase order handling (see Section 3) enriched with temporal constraints.

Based on the generated UPPAAL models, we performed the verification of the following CTL properties:
A[] not deadlock: to ensure deadlock freeness of the process,

A[] (Process.Delivergoods imply $t_{0} \leq 120$ ): to verify the process deadline is met.

Finally, the UPPAAL model checker proves the process model against the aforementioned CTL properties.

Actually, we assume that our verification approach is limited to the duration of activities (i.e. theduration constraint) and the time between events (i.e. the temporal dependency constraint).

Currently, we are working on introducing the notion of absolute time in timed automata. The goal is to achieve a clear relation between clocks of the process timed automaton in order to enrich the specification. And thus, enable a more efficient verification that facilitates the early discovery of temporal violations during process management life-cycle.

We aim to enhance the formal specification of processes by considering absolute time constraints as well as relative constraints

By using our verification process, every organization can verify the correctness of the temporal constraints of its processes.

\section{RELATED WORK}

Over the last decade, a number of research projects were conducted in an attempt to model temporal constraints in business process diagrams using the defacto industrial standard for business process modeling, BPMN [7, 6, 9, 3]. Indeed, the visual appeal of the graph-based modeling approaches makes them useful for all kinds of workflow designers since no technical background is required. Similarly, other research efforts $[14,13,11,5,10]$ opted for formal specification languages with modeling capabilities such as Petri nets and Timed Automata. Whereas, other approaches like [18] opted for CSP as a process algebra language, which lacks for graphical support. Table 9 presents an attempt to compare existing research approaches with regard to the supported temporal constraints.

In [12], the authors proposed time-patterns to foster selection of appropriate process aware information system. Although this work brings out interesting patterns, nevertheless our model supports larger set of temporal constraints.

Silver proposed in his blog [15] a BPMN extension to add 


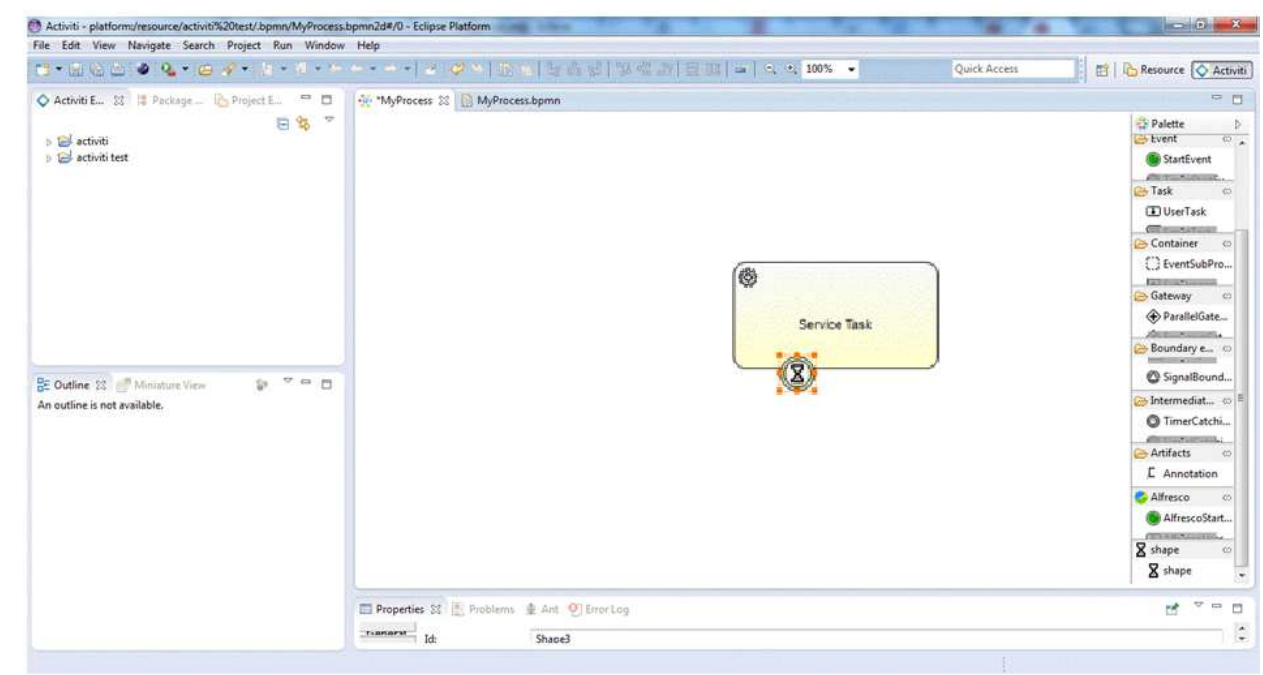

Figure 8: The screenshot of the prototype extending the Activiti Eclipse Designer

Table 9: A comparative table of the supported Temporal Constraints (TC) in the existing business process models

\begin{tabular}{|c|c|c|c|c|c|c|c|c|c|}
\hline & \multicolumn{3}{|c|}{ Intra-activity TC } & \multicolumn{3}{|c|}{ Inter-activity TC } & \multicolumn{3}{|c|}{ Inter-Processes TC } \\
\hline Approaches $[\mathrm{REF}]$ & 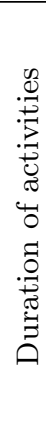 & 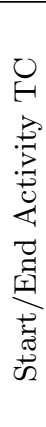 & 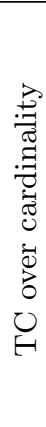 & 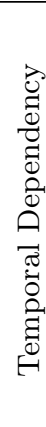 & 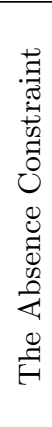 & 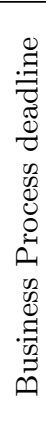 & 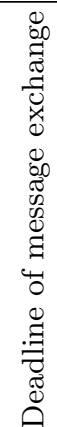 & 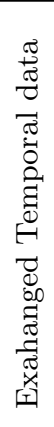 & 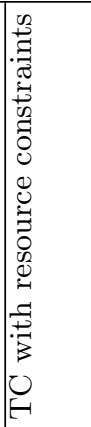 \\
\hline Gagne et al. [7] & & $\sqrt{ }$ & & $\sqrt{ }$ & & & & & \\
\hline Watahiki et al. [16] & $\sqrt{ }$ & & & & & & & & \\
\hline Huai et al. [10] & $\sqrt{ }$ & & & & & & $\sqrt{ }$ & & \\
\hline Du et al. [5] & $\sqrt{ }$ & & & & & & & & $\sqrt{ }$ \\
\hline Kallel et al. [11] & & & $\sqrt{ }$ & $\sqrt{ }$ & & & $\sqrt{ }$ & $\sqrt{ }$ & \\
\hline Makni et al. [14, 13] & $\sqrt{ }$ & & & $\sqrt{ }$ & & $\sqrt{ }$ & & & \\
\hline Guermouche et al. [9] & $\sqrt{ }$ & & & $\sqrt{ }$ & $\sqrt{ }$ & & $\sqrt{ }$ & & \\
\hline
\end{tabular}

temporal constraints to BPMN. Unfortunately, his proposal includes new flow objects linked by sequence flows, and thus is not a conformant extension to the current BPMN version. The key feature of our approach is to offer a BPMNcompliant extension(see the BPMN specification [2] p.8).

The major contribution of Time-BPMN [7], is the extension of the BPMN Notation [1] with a large set of required temporalities. This extension deals with additional temporal constraints and dependencies between business process activities. This extension does not permit to model temporal constraints relating to the duration of activities. This extension deals with the various temporal constraints and dependencies between activities or sub-processes within the same process and not between independent processes.

In contrast to Time-BPMN the authors in [6] used only the constructs proposed in the BPMN specification to model the already enumerated temporal constraints, the Time-BPMN extension [7], otherwise provides new activity decorators and new association links between them. Our approach improves and extends the work presented in $[7,6]$.

In our previous work [3], we review the state-of-art in a survey on time-aware business process modeling. We analyzed and compared existing approaches in respect to their ability to deal with time-related properties in the business processes modelling phase. The research conducted so far allowed us to identify temporal constraints that should be incorporated into BPMN and to set the basis for the development of a visual notation. Indeed, there is no comprehensive temporal modelling extension for BPMN that allows clear and consistent representation of temporal constraints and their attributes.

\section{CONCLUSION AND FURURE WORK}

In this paper, we exhibited the weakness of the defacto in- 
dustrial standard for process modelling, BPMN, to represent the time dimension of processes. We proposed thereafter a BPMN compliant extension to support the specification of a large set of rich temporal constraints beyond those illustrated in the litterature. We particularly presented how the temporal dimension can be specified at design time, through temporal constraints. We do not claim to provide an exhaustive list of extensions required for the modelling of any possible temporal constraint.

Subsequently, we have shown the added value of the proposed BPMN temporal extensions by means of its application to a variety of example processes. Finally, we relied on a model checking based verification appraoch to detect temporal violations. Particularly, we used the timed automata formalism and the UPPAAL model checker. Our verification framework aims to assist in mitigating risk and facilitates the early discovery of temporal violations during business process management life-cycle.

The BPMN extensions proposed throughout this paper, really help process performers understanding, sharing and improving knowledge on their business processes. In addition, compliance with the BPMN notation can increase the opportunity to share the process definition and execution knowledge between users of different business process management systems and thus between different organisations.

\section{ACKNOWLEDGMENTS}

Part of this work has been supported by FP7-ICT IMAGINE research and development project, co-funded by the European Commission under the Virtual Factories and Enterprises (FoF-ICT- 2011.7.3, Grant Agreement No: 285132).

\section{REFERENCES}

[1] Object Management Group (OMG), Business Process Modeling Notation (BPMN), Version 1.1, 2008.

[2] Object Management Group (OMG), Business Process Model and Notation (BPMN), Version 2.0, 2011.

[3] S. Cheikhrouhou, S. Kallel, N. Guermouche, and M. Jmaiel. A Survey on Time-aware Business Process Modeling. In Proceedings of the 15th International Conference on Enterprise Information Systems. SCITEPRESS, 2013.

[4] S. Cheikhrouhou, S. Kallel, N. Guermouche, and M. Jmaiel. Time-aware Automatic Process View Generation. In Proceedings of the 10th IEEE International Conference on e-Business Engineering. IEEE Computer Society, 2013.

[5] Y. Du, P. Xiong, Y. Fan, and X. Li. Dynamic Checking and Solution to Temporal Violations in Concurrent Workflow Processes. IEEE Transactions on Systems, Man, and Cybernetics, Part A, 41(6):1166-1181, 2011.

[6] C. Flores and M. Sepúlveda. Temporal Specification of Business Processes through Project Planning Tools. In Proceedings of the International Workshops and Education Track on Business Process Management Workshops (BPM), volume 66 of LNBIP, pages 85-96. Springer, 2010.

[7] D. Gagné and A. Trudel. Time-BPMN. In Proceedings of the IEEE Conference on Commerce and Enterprise Computing $(C E C)$, pages 361-367. IEEE Computer Society, 2009.
[8] N. Guermouche and C. Godart. Timed Conversational Protocol Based Approach for Web Services Analysis. In Proceedings of the 8th International Conference on Service-Oriented Computing, ICSOC, volume 6470 of LNCS, pages 603-611. Springer, 2010.

[9] N. Guermouche and S. D. Zilio. Towards Timed Requirement Verification for Service Choreographies. In Proceedings of the 8th IEEE International Conference on Collaborative Computing: Networking, Applications and Worksharing, page 10, USA, Oct. 2012.

[10] W. Huai, X. Liu, and H. Sun. Towards Trustworthy Composite Service Through Business Process Model Verification. In Proceedings of the 7th International Conference on Ubiquitous Intelligence \& Computing and 7th International Conference on Autonomic $8 \mathcal{G}$ Trusted Computing (UIC/ATC), pages $422-427$, 2010.

[11] S. Kallel, A. Charfi, T. Dinkelaker, M. Mezini, and M. Jmaiel. Specifying and Monitoring Temporal Properties in Web Services Compositions. In Proceedings of the 7th IEEE European Conference on Web Services, pages 148-157. IEEE Computer Society, 2009.

[12] A. Lanz, B. Weber, and M. Reichert. Workflow time patterns for process-aware information systems. In Enterprise, Business-Process and Information Systems Modeling, volume 50 of LNBIP, pages 94-107. Springer, 2010.

[13] M. Makni, N. B. Hadj-Alouane, S. Tata, and M. M. Yeddes. Negotiating Deadline Constraints in Inter-organizational Logistic Systems: A Healthcare Case Study. In Proceedings of the International Workshops and Education Track on Business Process Management Workshops (BPM), volume 100 of LNBIP, pages 108-118. Springer, 2011.

[14] M. Makni, S. Tata, M. M. Yeddes, and N. B. Hadj-Alouane. Satisfaction and Coherence of Deadline Constraints in Inter-Organizational Workflows. In Proceedings of the Confederated International Conferences: CoopIS on On the Move to Meaningful Internet Systems: OTM 2010, volume 6426 of LNCS, pages 523-539. Springer, 2010.

[15] B. Silver. My New BPMN Wish List, BPMS Watch Blog. Technical report, http://www.brsilver.com/wordpress/2008/07/17/mynew-bpmn-wish-list/, 2009.

[16] K. Watahiki, F. Ishikawa, and K. Hiraishi. Formal Verification of Business Processes with Temporal and Resource constraints. In Proceedings of the IEEE International Conference on Systems, Man and Cybernetics, pages 1173-1180. IEEE, 2011.

[17] P. Wohed, W. M. van der Aalst, M. Dumas, A. H. ter Hofstede, and N. Russell. On the suitability of bpmn for business process modelling. In Business Process Management, volume 4102 of $L N C S$, pages 161-176. Springer, 2006.

[18] P. Y. H. Wong and J. Gibbons. A Relative Timed Semantics for BPMN. Electronic Notes in Theoretical Computer Science, 229(2):59-75, 2009. 